\title{
Observational study comparing intranasal mometasone furoate with oral antihistamines for rhinitis and asthma
}

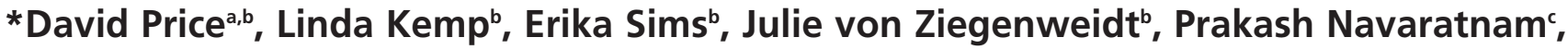 \\ Amanda J Lee ${ }^{d}$, Alison Chisholm ${ }^{b}$, Elizabeth V Hillyerb, Gokul Gopalan ${ }^{\text {e }}$
}

academic Centre of Primary Care, University of Aberdeen, Aberdeen, UK

${ }^{\mathrm{b}}$ Research in Real Life Ltd, Norwich, UK

' Global Outcomes Research, Merck \& Co., Inc., Whitehouse Station, NJ, US

${ }^{d}$ Medical Statistics Team, Section of Population Health, University of Aberdeen, Aberdeen, UK

e Global Medical Affairs Department, Merck \& Co., Inc., Whitehouse Station, NJ, US

Received 16th February 2010; revised 29th April 2010; accepted 17th May 2010; online 22nd June 2010

\begin{abstract}
Aims: Retrospective database study comparing upper and lower airway-related outcomes for patients with rhinitis and co-morbid asthma receiving mometasone furoate - an intranasal corticosteroid with low systemic bioavailability - or an oral antihistamine.

Methods: 395 patients prescribed intranasal mometasone were matched on 10 demographic and respiratory-related criteria in a 1:2 ratio to 790 patients prescribed oral antihistamine. Asthma and rhinitis control were assessed over one year using predefined composite proxy measures.

Results: Asthma control was achieved by $309 / 395$ (78.2\%) versus 580/790 (73.4\%; $p=0.071)$ patients in the mometasone and antihistamine cohorts, respectively. Rhinitis control was achieved by $293(74.2 \%)$ versus $539(68.2 \% ; p=0.035)$, respectively. The adjusted odds ratios for antihistamines, relative to mometasone, were 0.71 (95\% Cl, 0.52-0.98) for achieving asthma control and $0.74(95 \% \mathrm{Cl}$, 0.56-0.97) for achieving rhinitis control.

Conclusions: Patients with rhinitis and co-morbid asthma initiating rhinitis therapy achieved significantly better upper as well as lower airway outcomes with intranasal mometasone than with oral antihistamine.

(C) 2010 Primary Care Respiratory Society UK. All rights reserved.

D Price et al. Prim Care Resp J 2010; 19(3): 266-273

doi:10.4104/pcrj.2010.00040
\end{abstract}

Keywords antihistamine, asthma, control, mometasone furoate, one airway, rhinitis

The full version of this paper, with online Appendix

and supplementary data, is available at www.thepcrj.org

\section{Introduction}

Rhinitis imposes a substantial burden on patients and healthcare systems. Patients with rhinitis experience reduced quality of life and impaired physical and social functioning. ${ }^{1-4}$ Equally important, for patients with rhinitis and co-morbid asthma, there is increasing evidence that active rhinitis negatively affects asthma control. ${ }^{4-8}$ Patients with both rhinitis and asthma are more likely to be hospitalised, require urgent care, or visit their general practitioner (GP) for asthma than those with asthma alone.
Many patients have both rhinitis and asthma. One study found that approximately $50 \%$ of patients with allergic rhinitis to animals or mites had allergic asthma to the same triggers. ${ }^{9}$ Overall, an estimated $10-40 \%$ of patients with rhinitis have comorbid asthma. ${ }^{4,10,11}$ Moreover, bronchial hyperresponsiveness has been reported in patients who have rhinitis but no symptoms of asthma; conversely, segmental bronchial provocation can induce nasal inflammation in patients with rhinitis. ${ }^{12-14}$ The frequent association of asthma with rhinitis, the similar characteristics of inflammation in these two disorders, and the contiguous mucosal link between the upper and lower airways, all support the concept of the 'One Airway.'

The Allergic Rhinitis and its Impact on Asthma (ARIA) ${ }^{4}$ and

\footnotetext{
* Corresponding author: Professor David Price, Academic Centre of Primary Care, University of Aberdeen, Foresterhill Health Centre, Westburn Road, Aberdeen, AB25 2AY, UK. Tel: +44 1224554588 Fax: +44 1224550683 E-mail: david@respiratoryresearch.org
} 
primary care rhinitis guidelines ${ }^{5}$ recognise the 'One Airway' concept and emphasise the importance of treating both upper and lower airways in patients with co-morbid rhinitis and asthma. There is evidence that asthma exacerbations and hospitalisations are reduced in patients receiving intranasal corticosteroids. $^{15,16}$ Thus, it has been hypothesised that the administration of intranasal corticosteroids could improve outcomes in patients with co-morbid allergic rhinitis and asthma. ${ }^{4}$ Intranasal corticosteroids are considered the most effective therapy for allergic rhinitis; other options include antihistamines and the antileukotrienes. ${ }^{4,5}$

The primary objective of this observational study was to compare predefined clinical endpoints relating to the upper and lower airways for patients with co-morbid rhinitis and asthma who were initiated on rhinitis therapy with intranasal mometasone furoate or oral antihistamine. Mometasone furoate is an intranasal corticosteroid with low systemic bioavailability ${ }^{17}$ and was selected to minimise potential bias associated with the systemic effects of some intranasal corticosteroids.

\section{Methods}

\section{Study design and data source}

This was a retrospective, matched cohort study using the General Practice Research Database (GPRD), a large computerised primary care database (http://www.gprd.com/). The GPRD contains deidentified, longitudinal data from 3.6 million active medical records and 13 million records overall from over 450 subscribing practices throughout the UK. A practice-based quality marker, the up-to-standard date, is generated by the GPRD for each subscribing practice, and data subsequent to the practice up-tostandard date are considered to be acceptable, research quality, prospectively recorded data. The GPRD is well-validated and used frequently for medical and health research. ${ }^{18}$

This 2-year observational study comprised a baseline year preceding the date of a first prescription for rhinitis treatment (index date) and an outcome period of one year after the index date. The study period was from January 1st 1990 to June 30th 2007. Patients in the mometasone cohort were available for analysis from April 1997, when intranasal mometasone furoate became available by prescription in the UK.

Approval was given for the use of GPRD data for this study by the GPRD Independent Scientific Advisory Committee. Study design and analyses were conducted according to Standard Operating Procedures of the study group. ${ }^{19}$

\section{Patients}

Patients of any age with diagnostic codes for both rhinitis and asthma who initiated rhinitis treatment with a single prescription on the index date were eligible for inclusion in the study. Patients had to have up-to-standard data for at least one year before and one year after the index date. Patients were excluded if they received two or more prescriptions for rhinitis on the index date, or if their clinical record contained a code for chronic obstructive pulmonary disease (COPD) at any time, or a prescription for maintenance oral corticosteroid therapy during the baseline year.

We selected a matched case-control approach because of substantial baseline differences between the two treatment cohorts. Each patient receiving a first prescription for mometasone was individually matched to two patients receiving a first prescription for oral antihistamine to maximise statistical power and the number of possible matches simultaneously, whilst losing as few patients as possible. Several matching algorithms between the two cohorts were investigated (blind to study outcomes) to minimise baseline differences and ensure validity of the outcomes. We ultimately applied 10 matching criteria, including two demographic characteristics (age \pm 5 years and same sex) and a determination of asthma control, during the baseline year. The composite proxy measure for baseline asthma control was defined as:

1) no recorded hospital attendance for asthma;

2) no prescription for oral corticosteroids; and

3) no primary care consultation, hospital admission, or emergency department attendance for lower respiratory tract infection (LRTI) requiring antibiotics during the baseline year.

The remaining seven matching criteria captured the number of upper and lower respiratory events recorded during the baseline year, as depicted in the online Figure (see Appendix 1 at www.thepcrj.org). These included acute oral corticosteroid courses, rhinitis consultations, all ear, nose, and throat (ENT) events, and consultations for respiratory diagnoses. The listing of codes used for matching is available on request.

\section{Outcome measures}

The two primary outcome measures were composite measures serving as proxies for asthma control and rhinitis control during the outcome year. We defined asthma control a priori and in line with international asthma management guidelines, ${ }^{20}$ as requiring all of the following in the outcome year:

1) no recorded hospital attendance for asthma (neither admission nor attendance at the emergency department or outpatient department, or use of out-of-hours services)

2) no prescription for oral corticosteroids

3) no primary care consultation, hospital admission, or emergency department attendance for LRTI requiring antibiotics

4) average daily prescribed dose of $\leq 200 \mathrm{mcg}$ salbutamol or $\leq 500$ mcg terbutaline.

Rhinitis control was defined a priori as no change in rhinitis therapy together with no more than two consultations for rhinitis during the one-year outcome period after the index date.

We also examined the disaggregated measures comprising the composite definitions. Other secondary outcome measures included: short-acting $\beta_{2}$-agonist use (calculated as the number of reliever doses/day, based on the number of prescriptions); the 
numbers of GP consultations for upper airways disease (rhinitis); changes in upper airways treatment; total rhinitis-related events (total ENT events); GP consultations for asthma; hospital admissions for asthma; and hospital admissions for likely respiratory disease (hospital admissions for definite or suspected asthma plus definite respiratory hospital admissions).

\section{Statistical analyses}

Baseline characteristics of the mometasone furoate and oral antihistamine cohorts were compared using conditional logistic regression to identify residual confounders that were significantly different between the two cohorts at baseline. For binary variables, matched $\mathrm{p}$-values were derived from standard conditional logistic regression models. For continuous characteristics, the variables were modelled as predictors of cohort membership. Continuous variables that were highly skewed were categorised before conditional logistic regression and the $p$-value for the overall effect was reported.

The socioeconomic status score used in the analyses was that assigned to practices by the GPRD using the Index of Multiple Deprivation as a proxy measure (http://www. communities.gov.uk/communities/neighbourhoodrenewal/depri vation/). Respiratory and other confounding diagnoses including rhinitis, COPD, gastro-oesophageal reflux disease and cardiac disease, were expressed using the Charlson comorbidity index $(\mathrm{CCl})$, a weighted index that accounts for number and severity of comorbidities. ${ }^{21}$ The $\mathrm{CCl}$ was calculated for each patient using ICD-9 matching algorithms produced by CliniClue software (http://www.cliniclue.com/software).

Potential confounders examined during the baseline year included respiratory and allergy treatments, consultations or hospital attendance for asthma or other respiratory illness, and medications that might interfere with asthma control. A complete list of potential confounders is detailed in online Appendix 1.

Odds ratios for the dichotomised definitions of asthma and rhinitis control were calculated using conditional logistic regression, adjusting for covariates that were unbalanced at baseline. Secondary outcome variables were also analysed using conditional logistic regression.

Data were analysed using SPSS version 15/16 (SPSS, Chicago, IL, USA), STATA (StataCorp LP, College Station, TX, USA), and Microsoft Excel.

\section{Results}

A total of 395 patients prescribed intranasal mometasone furoate were matched in a $1: 2$ ratio to 790 patients prescribed an oral antihistamine for rhinitis on the index date (online Figure). As compared broadly with the unmatched population in terms of asthma-related parameters, the matched mometasone cohort had, on average, milder disease to enable matching with the antihistamine cohort, as depicted in Table 1. All patients had diagnostic codes for both rhinitis and asthma, as per the inclusion criteria, and prescriptions for one or more therapies for asthma were on record in the GPRD for 353/395 (89.4\%) and 728/790 $(92.2 \%)$ of patients in the matched mometasone and antihistamine cohorts, respectively.

Matched cohorts were evenly divided between men and women, and the mean age was 37 years (range 1-81 years; Table 2). A total of 129 patients, comprising $11 \%$ of the patient population, were aged 12 and younger. Other baseline characteristics were similar between the matched cohorts except for cardiac disease diagnosis, significantly more common in patients initiating antihistamines, and prescribed inhaled corticosteroid (ICS) daily dose, significantly lower in the antihistamine cohort (Table 2). Therefore, both variables were included as confounders in the subsequent regression analyses. In each cohort, $93 \%$ of patients met the matching criterion of baseline asthma control. Baseline values of the outcome measures are summarised by cohort in Table 3.

Table 1. Asthma-related parameters for unmatched and matched populations during the baseline year before patients with co-morbid rhinitis and asthma received a first prescription for rhinitis therapy, as recorded in the General Practice Research Database (GPRD).

Mometasone furoate cohort

Oral antihistamine cohort

\begin{tabular}{|c|c|c|c|c|}
\hline & Unmatched $(n=756)$ & Matched $(n=395)$ & Unmatched $(n=12,210)$ & Matched $(n=790)$ \\
\hline ICS prescription & $342(45.2)$ & $143(36.2)$ & $2892(23.7)$ & 209 (26.5) \\
\hline SABA prescription & $459(60.7)$ & $203(51.4)$ & $4628(37.9)$ & $316(40.0)$ \\
\hline OCS prescription & $76(10.1)$ & $4(1.0)$ & $745(6.1)$ & $8(1.0)$ \\
\hline Asthma exacerbation* & $153(20.2)$ & $28(7.1)$ & $1953(16.0)$ & $56(7.1)$ \\
\hline
\end{tabular}


Mometasone vs antihistamines for rhinitis and asthma

Table 2. Demographic and clinical characteristics of matched cohorts.

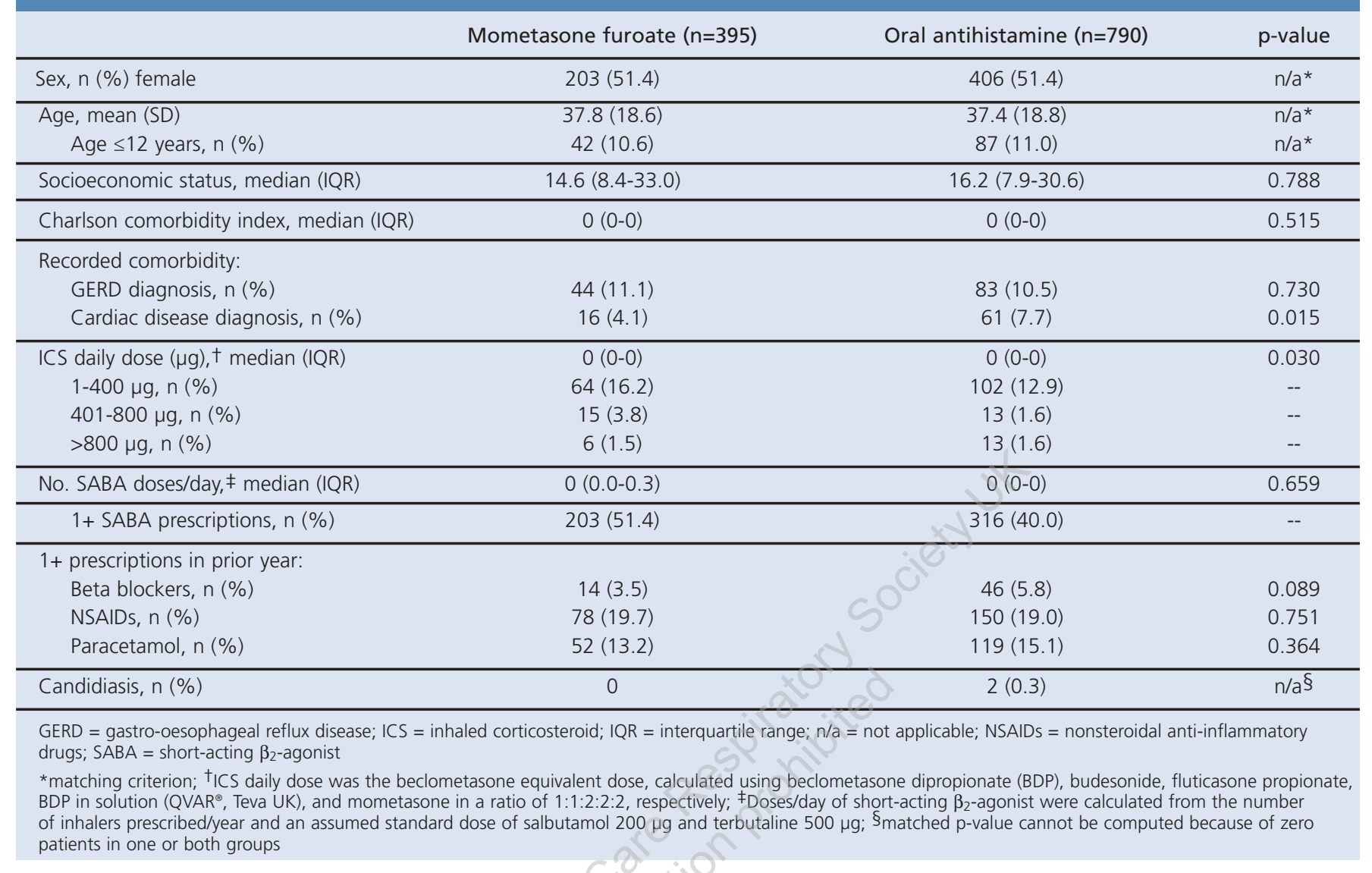

Most patients $(706 / 790,89 \%)$ in the antihistamine cohort were prescribed a second- or third-generation (non-sedating) antihistamine; $84(11 \%)$ were prescribed a first-generation antihistamine. One patient in each cohort had a prescription for an antileukotriene recorded during the baseline year.

\section{Outcomes}

The primary outcome of asthma control was achieved by three quarters of patients overall, including 309/395 (78.2\%) patients in the mometasone cohort and $580 / 790(73.4 \%)$ of those in the antihistamine cohort $(\mathrm{p}=0.071)$. After adjusting for residual confounding factors, the odds ratio for asthma control was significantly less for the antihistamine cohort than the mometasone cohort $(0.71$ [95\% Cl, 0.52-0.98]; Figure 1). Following disaggregation of the primary outcome variable, there appeared to be no single clinical variable driving the outcome of asthma control (Table 4).

Rhinitis control was achieved by significantly more patients receiving mometasone than antihistamine (293 [74.2\%] vs. $539[68.2 \%] ; p=0.035)$. The adjusted odds ratio for rhinitis control for patients receiving antihistamine, as compared with mometasone, was $0.74(95 \% \mathrm{Cl}, 0.56-0.97$; Figure 1). The main driver of cohort differences in rhinitis control was change in therapy, as a significantly smaller proportion $(p=0.022)$ of mometasone patients had a change in therapy during the
Figure 1. Adjusted odds ratios $(95 \% \mathrm{Cl})$ for asthma control (open circle, top bar) and rhinitis control (closed circle, bottom bar) for the oral antihistamine cohort, with intranasal mometasone furoate cohort as the comparator (odds ratio $=\mathbf{1 . 0}$ ).

*Odds ratios adjusted for baseline cardiac disease diagnosis and inhaled corticosteroid dose (categorised as $0 \mu \mathrm{g},>0-200 \mu \mathrm{g},>200-$ $400 \mu \mathrm{g},>\mathbf{4 0 0} \mu \mathrm{g}$ ). Asthma control was defined as no recorded hospital attendance for asthma, oral corticosteroids, or antibiotics for lower respiratory tract infection, plus an average daily prescribed dose of $\leq \mathbf{2 0 0} \boldsymbol{\mu g}$ salbutamol. Rhinitis control was defined as no change in rhinitis therapy together with no more than two consultations for rhinitis during the 1-year outcome period after the index date.

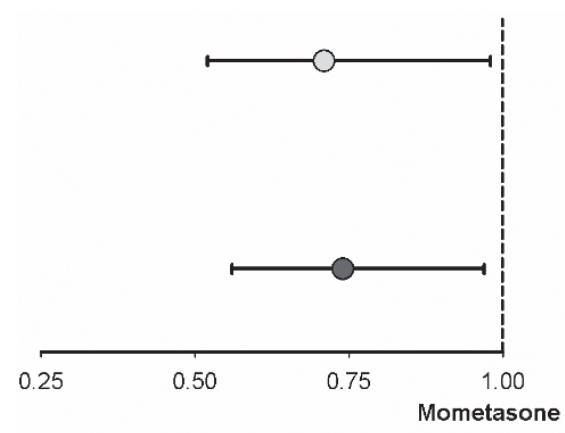

Asthma and rhinitis control (adjusted odds ratio*) 
D Price et al.

\section{Table 3. Baseline values of outcome measures for the two cohorts.}

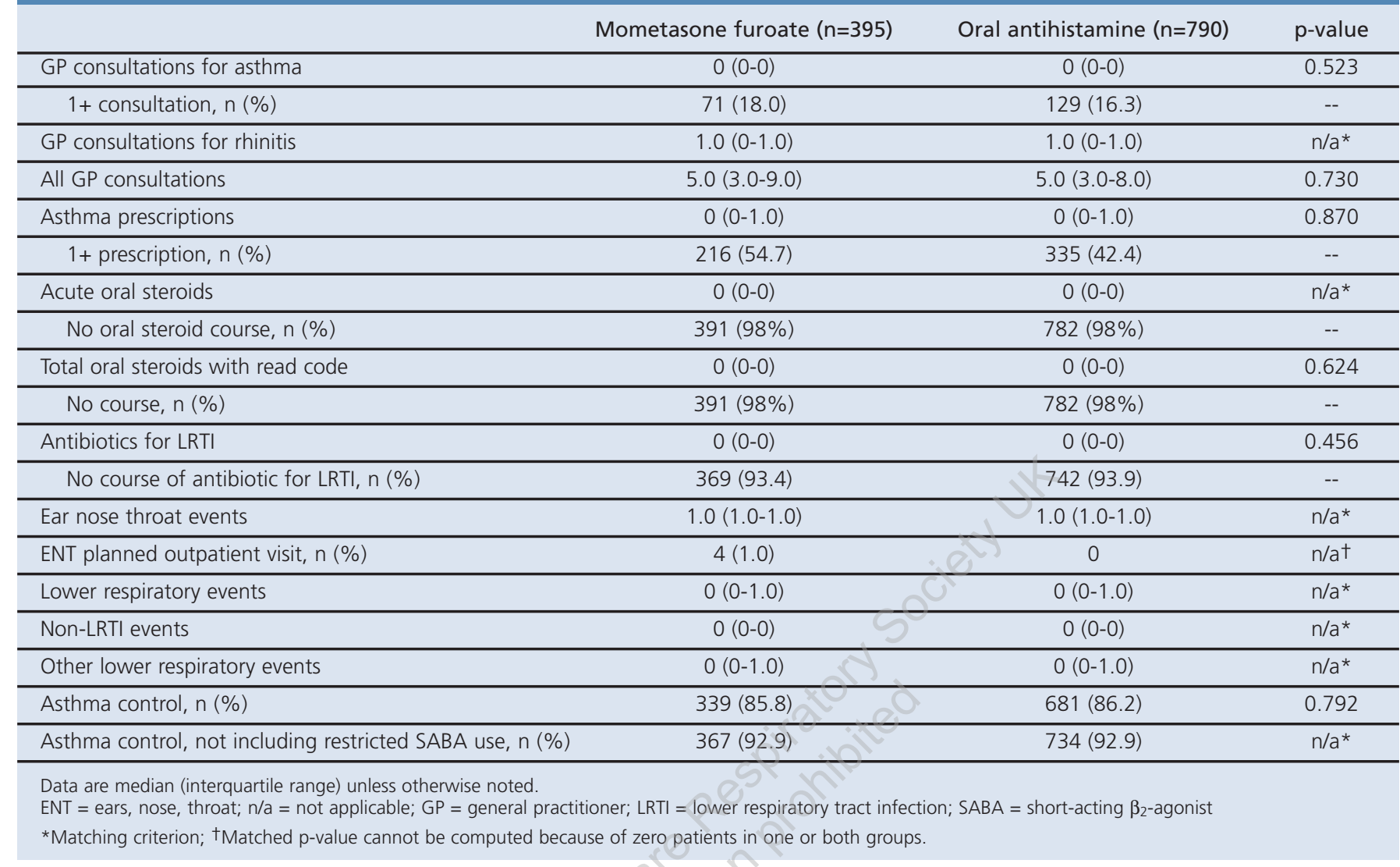

Table 4. Results for disaggregated primary outcome measures and secondary outcome measures during the outcome year. Outcome Measure Mometasone furoate $(n=395)$ Oral antihistamine $(n=790) \quad p$-value Disaggregated primary outcomes:

\begin{tabular}{|c|c|c|c|}
\hline No hospitalisation or hospital visit for asthma, $\mathrm{n}(\%)$ & $393(99.5)$ & $790(100)$ & $n / a^{*}$ \\
\hline No oral corticosteroid course, $\mathrm{n}(\%)$ & $377(95.4)$ & $740(93.7)$ & 0.214 \\
\hline No consultation for LRTI requiring antibiotics, n (\%) & $357(90.4)$ & $700(88.6)$ & 0.339 \\
\hline Salbutamol daily dose $\leq 200 \mu \mathrm{g} /$ day, ${ }^{\dagger} \mathrm{n}(\%)$ & $346(87.6)$ & $681(86.2)$ & 0.487 \\
\hline No change in rhinitis therapy, $\mathrm{n}(\%)$ & $296(74.9)$ & $541(68.5)$ & 0.022 \\
\hline Rhinitis consultations $\leq 2 / \mathrm{yr}, \mathrm{n}(\%)$ & $387(98.0)$ & $778(98.5)$ & 0.518 \\
\hline \multicolumn{4}{|l|}{ Secondary outcome measures: } \\
\hline No. SABA doses/day, $\neq$ median (IQR) & $0.0(0.00-0.55)$ & $0.0(0.00-0.55)$ & 0.592 \\
\hline $1+$ SABA prescriptions during the year, $\mathrm{n}(\%)$ & $167(42.3)$ & $321(40.6)$ & 0.567 \\
\hline GP consultations for asthma, median (IQR) & $0.0(0.0-1.0)$ & $0.0(0.0-0.0)$ & 0.900 \\
\hline 1+ GP consultation for asthma, n (\%) & $100(25.3)$ & $193(24.4)$ & 0.733 \\
\hline Hospitalisation (inpatient) for asthma, n (\%) & 0 & 0 & $n / a^{*}$ \\
\hline Hospitalisation for likely respiratory disease, n (\%) & $1(0.3)$ & $2(0.3)$ & 1.00 \\
\hline GP consultations for rhinitis, median (IQR) & $0.0(0.0-1.0)$ & $0.0(0.0-0.0)$ & 0.044 \\
\hline 1+ GP consultation for rhinitis, $\mathrm{n}(\%)$ & $102(25.8)$ & $156(19.7)$ & 0.016 \\
\hline Total ENT-related events, median (IQR) & $0.0(0.0-1.0)$ & $0.0(0.0-1.0)$ & 0.251 \\
\hline 1+ GP consultation for ENT, $n(\%)$ & $169(42.8)$ & $327(41.4)$ & 0.641 \\
\hline
\end{tabular}

$\mathrm{ENT}=$ ears, nose, throat; GP = general practitioner; IQR = interquartile range; LRTI = lower respiratory tract infection; SABA = short-acting $\beta_{2}$-agonist

* Matched p-value cannot be computed because of zero patients in one or both groups; †Or terbutaline $\leq 500 \mu \mathrm{g} / \mathrm{day}$; $\neq$ One dose of SABA was defined as salbutamol $200 \mu \mathrm{g}$ or terbutaline $500 \mu \mathrm{g}$. p-values from conditional logistic regression. 


\begin{tabular}{|c|c|c|c|c|c|c|c|c|}
\hline \multirow[b]{3}{*}{ First change class* } & \multicolumn{4}{|c|}{ Mometasone furoate } & \multicolumn{4}{|c|}{ Oral antihistamine } \\
\hline & \multirow[b]{2}{*}{$\begin{array}{l}\text { Single } \\
\text { change }\end{array}$} & \multicolumn{2}{|c|}{ Second change class* } & \multirow[b]{2}{*}{ Total } & \multirow[b]{2}{*}{$\begin{array}{l}\text { Single } \\
\text { change }\end{array}$} & \multicolumn{2}{|c|}{ Second change class* } & \multirow[b]{2}{*}{ Total } \\
\hline & & $\begin{array}{c}\text { Nasal } \\
\text { decongestant }\end{array}$ & $\begin{array}{c}\text { Oral } \\
\text { antihistamine }\end{array}$ & & & $\begin{array}{c}\text { Nasal } \\
\text { decongestant }\end{array}$ & $\begin{array}{c}\text { Oral } \\
\text { antihistamine }\end{array}$ & \\
\hline Mometasone & N/A & N/A & N/A & & 18 & 0 & 2 & 20 \\
\hline Nasal decongestant & 9 & 0 & 0 & 9 & 6 & 0 & 0 & 6 \\
\hline Nasal corticosteroid & 30 & 0 & 5 & 35 & 113 & 1 & 10 & 124 \\
\hline Oral antihistamine & 51 & 0 & 0 & 51 & 87 & 0 & 0 & 87 \\
\hline Oral decongestant & 4 & 0 & 0 & 4 & 12 & 0 & 0 & 12 \\
\hline Total & 94 & 0 & 5 & 99 & 236 & 1 & 12 & 249 \\
\hline
\end{tabular}

*Possible class types were mometasone, nasal decongestant, nasal corticosteroid, oral antihistamine, oral decongestant, nasal antihistamine, nasal saline

1-year outcome period (Table 4).

There were no significant differences between cohorts in short-acting $\beta_{2}$-agonist prescriptions or asthma-related events (Table 4).

Patients in the mometasone cohort had significantly more rhinitis consultations, whilst patients in the antihistamine cohort had significantly more changes in rhinitis therapy (Table 4). Of the patients with a treatment change, most in the mometasone cohort received an oral antihistamine or intranasal corticosteroid, while most in the antihistamine cohort received an intranasal corticosteroid. Table 5 summarises the treatment changes in each cohort during the outcome year. There was no significant difference between cohorts for ENT events overall during the outcome period (Table 4).

\section{Discussion}

Both asthma control and rhinitis control were significantly more likely in UK primary care for patients with rhinitis and co-morbid asthma who received intranasal mometasone furoate rather than an oral antihistamine as their first rhinitis therapy. We found that patients in the mometasone cohort had more GP consultations for rhinitis, yet a greater proportion of patients in the antihistamine cohort had a change in their rhinitis treatment, the latter driving the difference between cohorts in rhinitis control.

We used composite proxy measures to identify asthma and rhinitis control from the database records. The use of composite measures is recommended for asthma clinical trials to provide more complete assessment of asthma control than is possible by using a single measure.22 Their use is also pertinent for a retrospective, observational database study in which the measures recorded - coded diagnoses and medical resource use - serve as substitutes for direct clinical assessments. Indeed, there was not one single asthma-related factor driving the difference between cohorts in asthma control.

The composite for asthma control included measures that are recommended to define asthma exacerbations (hospitalisations, unscheduled use of secondary care, and oral corticosteroid courses), ${ }^{20,22,23}$ as well as prescriptions for antibiotics for LRTI, since the symptoms of an asthma exacerbation may be confused for those of respiratory infection. In addition, to maximise the power of the composite measure, we assessed the use of short-acting $\beta_{2}$-agonist, captured via prescribing records, as use of reliever therapy reflects the presence of asthma symptoms. . $22,23^{2}$

The criteria to define rhinitis control included no change in rhinitis treatment, which served as an indicator that no additional or different treatment was needed, and two consultations or fewer for rhinitis during the outcome year. We allowed for up to two scheduled visits on the basis that good practice would dictate at least one routine follow-up consultation after initiation of rhinitis treatment in addition to one longer-term review. The better odds of rhinitis control with mometasone versus oral antihistamine therapy is not unexpected, since the efficacy of intranasal corticosteroids in allergic rhinitis is well-established. ${ }^{4,24}$

We chose intranasal mometasone furoate as the comparator in this study, as the systemic effects of earlier intranasal corticosteroid formulations are considered a possible factor influencing lower respiratory outcomes in prior studies. ${ }^{10,25}$ Mometasone furoate is a topically active, synthetic corticosteroid lacking systemic effects that could influence asthma control. ${ }^{17}$ It would have been of interest to study other intranasal corticosteroids with low systemic bioavailability such as intranasal fluticasone and ciclesonide; and this is a topic for future study.

Patients in the GPRD who were prescribed mometasone tended to have worse asthma symptoms than those prescribed antihistamines. A challenge in designing this study was that, to enable matching with the antihistamine cohort, we had to limit our analyses to the mometasone-treated patients with milder asthma. Thus, our study results may underestimate the effects of mometasone because of ceiling effects in these patients with relatively mild disease. More severe rhinitis is typically associated with worse asthma control. 4,26 Nonetheless, as allergic rhinitis and asthma are highly prevalent conditions, and both negatively 
impact on health-related quality of life, ${ }^{4}$ even a modest improvement in asthma symptoms is important.

Two recent prospective studies report no additional effect of rhinitis therapy on co-morbid asthma symptoms, possibly because of little room for further improvement in lung function. In the first of these, ${ }^{25}$ improvements in lower airway function were not evident for patients with seasonal rhinitis and asthma receiving only intranasal fluticasone and, conversely, improvements in nasal symptoms were not evident for those receiving only inhaled fluticasone. These findings led the authors to conclude that the combination of ICS and intranasal corticosteroids is optimal for these patients. ${ }^{25}$ In the second study, $^{27}$ therapy with intranasal fluticasone conferred no additional benefit in overall asthma control for patients with comorbid rhinitis and asthma who were also receiving inhaled fluticasone propionate and salmeterol, possibly because the lower airways were already effectively treated. Moreover, the length of the aforementioned studies (6 and 4 weeks, respectively) was insufficient to capture less frequent evidence of uncontrolled asthma, such as exacerbations. In a small, 4-month placebo-controlled study, improvements in asthma symptoms and degree of bronchial responsiveness to histamine were not evident until 75 days after initiation of intranasal beclometasone. ${ }^{28}$

Nair and coworkers ${ }^{29}$ reported recently that intranasal fluticasone had no statistically significant steroid-sparing effect on ICS dose for patients with persistent allergic rhinitis and asthma, as determined by bronchial methacholine challenge after two weeks of treatment. The 25 patients studied had demonstrated airway hyper-responsiveness (methacholine PC $20_{20}$ $<4 \mathrm{mg} / \mathrm{ml}$ ), no steroid responsiveness, and the study may have been somewhat underpowered having $80 \%$ power to detect one doubling-dilution shift in methacholine $\mathrm{PC}_{20}$. Of note, there was no significant difference demonstrated in the study for low dose ICS plus intranasal corticosteroids compared with high dose ICS. Furthermore, the authors note a need for long-term studies to examine effect of combined therapy on exacerbations, which was part of the composite outcome measure of asthma control used in this study. ${ }^{29}$

Prior retrospective cohort studies have reported reduced asthma-related hospitalisations and asthma exacerbations for patients with asthma receiving therapy for their allergic rhinitis. ${ }^{15,16}$ By contrast, a third observational study found no benefit of intranasal corticosteroids in preventing asthma-related hospitalisations. ${ }^{30}$ Confounding by indication may be one possible explanation for this finding, whereby the true effect of intranasal corticosteroids is underestimated because treatments are not randomly assigned and it is the patients with more severe asthma who are prescribed intranasal corticosteroids. This difference was evident (although not statistically tested) in asthma-related parameters between the unmatched cohorts at

\section{Summary box}

Difficulties encountered during this study: Substantial baseline differences between the two treatment cohorts, leading to matched case-control approach. Inconsistent coding for "allergic rhinitis" in the database, leading to use of the broader definition of "rhinitis"

Alternative method that would be helpful in answering the research question: Prospective observational or randomised trial in primary care.

Lessons for clinical practice as a result of the study: Patients with rhinitis and co-morbid asthma initiating rhinitis therapy achieve better upper and lower airway outcomes with intranasal mometasone than with oral antihistamine. Study results support the approach of primary care and ARIA guidelines in recommending concomitant treatment of upper and lower airways for patients with co-morbid rhinitis and asthma.

baseline in the present study. We therefore attempted to minimise this effect by matching patients at baseline for multiple characteristics, including asthma control.

By restricting the analysis to newly treated patients we avoided the immortal time bias characterising a previous observational study in rhinitis. ${ }^{30,31}$ Moreover, the study of outcomes over a full year after the index prescription minimised the influence of seasonal variations in symptoms. While we attempted to address all confounding factors, a limitation of this study, as with all observational studies, is the possibility of unrecognised confounding factors. The accuracy of the diagnostic and treatment codes in the database relies on the accuracy of coding at each practice subscribing to the GPRD. As the coding for "allergic rhinitis" was inconsistent in the database, we studied patients with a diagnostic code for the broader definition of "rhinitis"; therefore, it is possible that some patients had non-allergic rhinitis. In addition, there is no guarantee that patients actually took the treatments as prescribed. Finally, we were unable to capture over-the-counter drug purchases and thus were unable to account for patient selftreatment. Therefore, it is important that our results be assessed in conjunction with those of other studies, both observational and randomised controlled trials.

\section{Conclusion}

This study has allowed us to examine real world results of rhinitis therapy in an unselected UK primary care patient population. We found that patients with rhinitis and comorbid asthma prescribed intranasal mometasone furoate as their first rhinitis therapy achieved significantly better outcomes - with regard to both asthma and rhinitis control when compared with patients prescribed oral antihistamines. Our findings support the approach of primary care and ARIA guidelines in recommending concomitant treatment of upper and lower airways for patients with co-morbid rhinitis and 
asthma. More prospective studies of diverse patients in primary care are needed to support and extend our findings.

\section{Conflict of interest declaration}

David Price has consultant arrangements with Aerocrine, Boehringer Ingelheim, Dey Pharmaceuticals, GlaxoSmithKline, Merck, Sharpe and Dohme, Novartis, ScheringPlough, and Teva. He or his team have received grants and research support for research in respiratory disease from the following organisations: UK National Health Service, Aerocrine, AstraZeneca, Boehringer Ingelheim, GlaxoSmithKline, Merck, Sharpe and Dohme, Novartis, Pfizer, Schering Plough, and Teva. He has spoken for Boehringer Ingelheim, GlaxoSmithKline, Merck, Sharpe and Dohme, Pfizer, and Teva.

Linda Kemp has no conflict of interest to declare.

Erika Sims has worked on projects funded by Schering Plough, Merck \& Co., Inc., and Teva, and has received funding to attend conferences.

Julie von Ziegenweidt has no conflict of interest to declare.

Prakash Navaratnam has a consultant arrangement with Merck \& Co., Inc. He is the CEO of Eympres Research LLC, Hilliard, Ohio, USA.

Amanda J. Lee receives payment for statistical consultancy through the independent research company, Thorpe Respiratory Research.

Alison Chisholm has no conflict of interest to declare.

Elizabeth V. Hillyer has done freelance writing work for Merck \& Co., Inc., Aerocrine, and Teva Santé and received reimbursement for work-related travel expenses from Thorpe Respiratory Research and Merck.

Gokul Gopalan is an employee of Merck \& Co., Inc.

\section{References}

1. Canonica GW, Bousquet J, Mullol J, Scadding GK, Virchow JC. A survey of the burden of allergic rhinitis in Europe. Allergy 2007;62 Suppl 85:17-25. http://dx.doi.org/10.1111/j.1398-9995.2007.01549.x

2. Schatz M. A survey of the burden of allergic rhinitis in the USA. Allergy 2007;62 Suppl 85:9-16. http://dx.doi.org/10.1111/j.1398-9995.2007.01548.x

3. Simoens $\mathrm{S}$, Laekeman $\mathrm{G}$. Pharmacotherapy of allergic rhinitis: a pharmaco-economic approach. Allergy 2009;64(1):85-95. http://dx.doi.org/10.1111//.113989995.2008.01909.x

4. Bousquet J, Khaltaev N, Cruz AA, et al. Allergic Rhinitis and its Impact on Asthma (ARIA) 2008 update (in collaboration with the World Health Organization, GA(2)LEN and AllerGen). Allergy 2008;63 Suppl 86:8-160. http://dx.doi.org/10.1111/j.13989995.2007.01620.x

5. Price D, Bond C, Bouchard J, et al. International Primary Care Respiratory Group (IPCRG) Guidelines: management of allergic rhinitis. Prim Care Resp J 2006;15(1):58-70. http://dx.doi.org/10.1016/j.pcrj.2005.11.002

6. Price D, Zhang Q, Kocevar VS, Yin DD, Thomas M. Effect of a concomitant diagnosis of allergic rhinitis on asthma-related health care use by adults. Clin Exp Allergy 2005;35(3):282-7. http://dx.doi.org/10.1111/j.1365-2222.2005.02182.x

7. Thomas $M$, Kocevar VS, Zhang Q, Yin DD, Price D. Asthma-related health care resource use among asthmatic children with and without concomitant allergic rhinitis. Pediatrics 2005;115(1):129-34. http://dx.doi.org/10.1542/peds.2004-0067

8. Magnan A, Meunier JP, Saugnac C, Gasteau J, Neukirch F. Frequency and impact of allergic rhinitis in asthma patients in everyday general medical practice: a French observational cross-sectional study. Allergy 2008;63(3):292-8. http://dx.doi.org/ 10.1111/j.1398-9995.2007.01584.x

9. Linneberg A, Henrik Nielsen N, Frolund L, Madsen F, Dirksen A, Jorgensen T. The link between allergic rhinitis and allergic asthma: a prospective population-based study. The Copenhagen Allergy Study. Allergy 2002;57(11):1048-52.

10. Thomas M. Allergic rhinitis: evidence for impact on asthma. BMC Pulm Med 2006;6 Suppl 1:S4. http://dx.doi.org/10.1186/1471-2466-6-S1-S4

11. Leynaert B, Neukirch $C$, Kony $S$, et al. Association between asthma and rhinitis according to atopic sensitization in a population-based study. J Allergy Clin Immunol 2004;113(1):86-93. http://dx.doi.org/10.1016/j.jaci.2003.10.010

12. Boulet LP. Asymptomatic airway hyperresponsiveness: a curiosity or an opportunity to prevent asthma? Am J Respir Crit Care Med 2003;167(3):371-8. http://dx.doi.org/10.1164/rccm.200111-084PP
13. Braunstahl GJ, Kleinjan A, Overbeek SE, Prins JB, Hoogsteden HC, Fokkens WJ. Segmental bronchial provocation induces nasal inflammation in allergic rhinitis patients. Am J Respir Crit Care Med 2000;161(6):2051-7.

14. Ciprandi G, Cirillo I, Pistorio A. Impact of allergic rhinitis on asthma: effects on spirometric parameters. Allergy 2008;63(3):255-60. http://dx.doi.org/10.1111/ j.1398-9995.2007.01544.x

15. Corren J, Manning BE, Thompson SF, Hennessy S, Strom BL. Rhinitis therapy and the prevention of hospital care for asthma: a case-control study. J Allergy Clin Immunol 2004;113(3):415-9. http://dx.doi.org/10.1016/j.jaci.2003.11.034

16. Crystal-Peters J, Neslusan C, Crown WH, Torres A. Treating allergic rhinitis in patients with comorbid asthma: the risk of asthma-related hospitalizations and emergency department visits. J Allergy Clin Immunol 2002;109(1):57-62.

17. Penagos M, Compalati E, Tarantini F, Baena-Cagnani CE, Passalacqua G, Canonica GW. Efficacy of mometasone furoate nasal spray in the treatment of allergic rhinitis. Meta-analysis of randomized, double-blind, placebo-controlled, clinical trials. Allergy 2008;63(10):1280-91. http://dx.doi.org/10.1111/j.1398-9995. 2008.01808.x

18. Lawson DH, Sherman V, Hollowell J. The General Practice Research Database. Scientific and Ethical Advisory Group. QJM 1998;91(6):445-52.

19. Research in Real Life, Ltd. Standard Operating Procedure. Available from: http://www.optimumpatientcare.org/downloads/documents/SOP\%20observational $\% 20$ database\%20studies.pdf.

20. Global Initiative for Asthma (GINA). Global Strategy for Asthma Management and Prevention, updated 2009. Available from: http://www. ginasthma.org.

21. Charlson ME, Pompei P, Ales KL, MacKenzie CR. A new method of classifying prognostic comorbidity in longitudinal studies: development and validation. J Chronic Dis 1987; 40(5):373-83.

22. Reddel HK, Taylor DR, Bateman ED, et al. An official American Thoracic Society/European Respiratory Society statement: Asthma control and exacerbations: standardizing endpoints for clinical asthma trials and clinical practice. Am J Respir Crit Care Med 2009;180(1):59-99. http://dx.doi.org/ 10.1164/rccm.200801-060ST

23. Thomas M, von Ziegenweidt J, Lee AJ, Price D. High-dose inhaled corticosteroids versus add-on long-acting beta-agonists in asthma: an observational study. J Allergy Clin Immunol 2009;123(1):116-21 e10. http://dx.doi.org/10.1016/ j.jaci.2008.09.035

24. Weiner JM, Abramson MJ, Puy RM. Intranasal corticosteroids versus oral $\mathrm{H} 1$ receptor antagonists in allergic rhinitis: systematic review of randomised controlled trials. BMJ 1998;317(7173):1624-9.

25. Dahl R, Nielsen LP, Kips J, et al. Intranasal and inhaled fluticasone propionate for pollen-induced rhinitis and asthma. Allergy 2005;60(7):875-81. http://dx.doi.org/10.1111/j.1398-9995.2005.00819.x

26. Togias A. Rhinitis and asthma: evidence for respiratory system integration. J Allergy Clin Immunol 2003;111(6):1171-83.

27. Nathan RA, Yancey SW, Waitkus-Edwards K, et al. Fluticasone propionate nasal spray is superior to montelukast for allergic rhinitis while neither affects overall asthma control. Chest 2005;128(4):1910-20. http://dx.doi.org/10.1378/ chest.128.4.1910

28. Agondi RC, Machado ML, Kalil J, Giavina-Bianchi P. Intranasal corticosteroid administration reduces nonspecific bronchial hyperresponsiveness and improves asthma symptoms. J Asthma 2008;45(9):754-7. http://dx.doi.org/10.1080/ 02770900802249149

29. Nair A, Vaidyanathan S, Clearie K, Williamson P, Meldrum K, Lipworth BJ. Steroid sparing effects of intranasal corticosteroids in asthma and allergic rhinitis. Allergy 2010;65(3):359-67. http://dx.doi.org/10.1111/j.1398-9995.2009.02187.x

30. Suissa S, Ernst P. Bias in observational study of the effectiveness of nasal corticosteroids in asthma. J Allergy Clin Immunol 2005;115(4):714-19. http://dx.doi.org/10.1016/j.jaci.2004.12.1118

31. Adams RJ, Fuhlbrigge AL, Finkelstein JA, Weiss ST. Intranasal steroids and the risk of emergency department visits for asthma. J Allergy Clin Immunol 2002;109(4):63642. 
Mometasone vs antihistamines for rhinitis and asthma

Appendix 1 . Flow diagram showing patient identification and cohort matching from the GPRD.

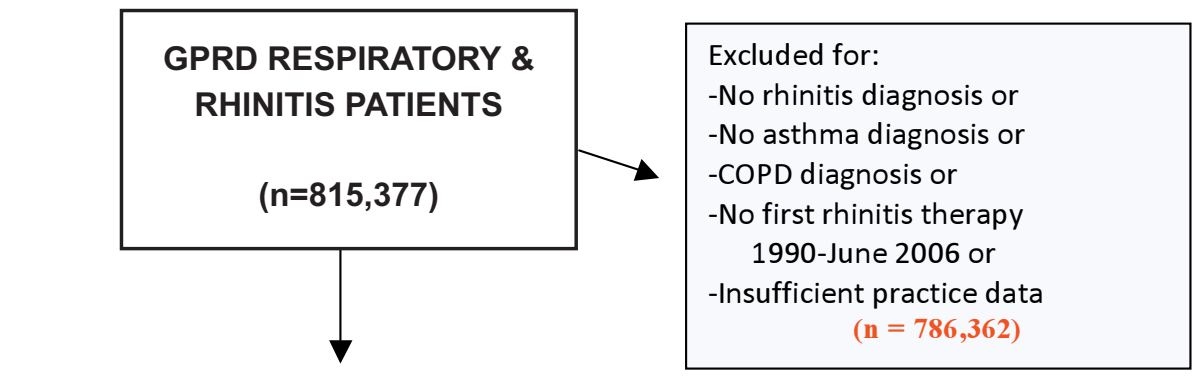

Patients initiating rhinitis therapy \& meeting all inclusion/exclusion criteria $(n=29,015)$

Match Mometasone Patients to Oral Antihistamine on the following criteria:

Patients prescribed mometasone ( $n=756$ )
Patients prescribed oral antihistamine $(n=12,210)$

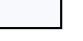

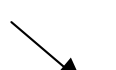

Lost on Matching for age:

Mometasone $(n=0)$

Antihistamine $(n=0)$

Lost on Matching for control:

Mometasone $(n=0)$

Antihistamine $(n=1)$

Lost on Matching for rhinitis consult: Mometasone $(n=4)$

Antihistamine $(n=208)$

Lost on Matching for oral steroid: Mometasone $(n=2)$

Antihistamine $(n=204)$

Lost on Matching for lower resp:

Mometasone $(n=10)$

Antihistamine $(n=649)$ 
D Price et al.

Appendix 1 . Flow diagram showing patient identification and cohort matching from the GPRD continued.

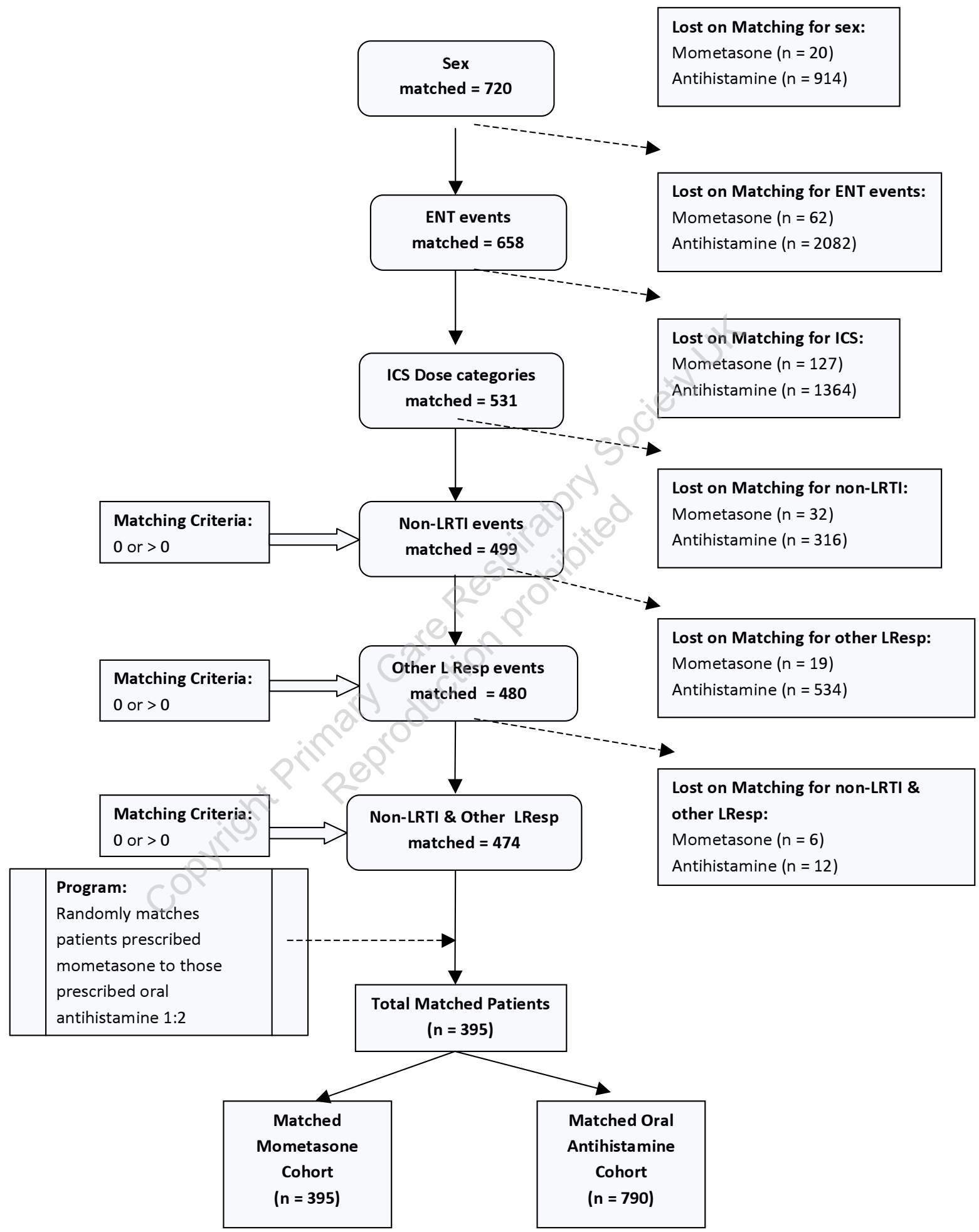

ENT, ears, nose, throat; GPRD, General Practice Research Database; ICS, inhaled corticosteroid; LResp, lower respiratory; LRTI, lower respiratory tract infection 


\section{Appendix 1. Potential confounders examined for the two cohorts}

The following potential confounders were examined at or closest to the relevant index date:

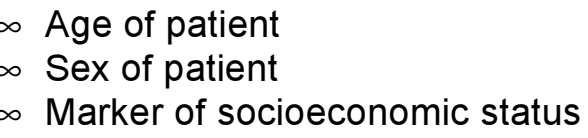

The following potential confounders were examined over all times (ever):

$\infty$ Other respiratory or other confounding diagnoses including rhinitis, chronic obstructive pulmonary disease (COPD), gastro-oesophageal reflux disease, and cardiac disease. These were expressed using the Charlson comorbidity index. ${ }^{1}$

The following potential confounders were examined in the year before the index date:

All respiratory, allergy, and other respiratory treatments

Prescribed inhaled corticosteroid (ICS) daily dose in beclometasone equivalents. Daily dose of ICS is the average number of puffs patient has been taking per day calculated from number of inhalers prescribed multiplied by doses per pack over the year.

Number of courses of oral corticosteroids

Number of general practice consultations for asthma or other respiratory illness

Number of hospital outpatient attendances where asthma is recorded as the reason for referral

Number of hospitalisations for asthma or possibly respiratory related

Number of prescriptions for any antibiotic where the reason for the prescription is RTI, LRTI, or URTI.

Asthma control status

Other medications that might interfere with asthma control: beta blockers, NSAIDs and paracetamol

Candidiasis

ENT events

Prescriptions for theophylline, long-acting $\beta 2$-agonist, leukotriene receptor antagonist SABA dosage/daily dose/inhalers/prescriptions

\section{Code lists}

Code lists, based on OXMIS, Read and drug codes lists, were developed by the researchers, who include part-time academic GPs (available on request). These have been developed and refined over the last 3 years in their own research and in collaboration with other academic partners in a large number of primary care database studies. Those making up the Charlson comorbidity index have been developed using ICD-9 matching algorithms produced by CliniClue ${ }^{\circledR}$ (www.cliniclue.com).

1. Charlson ME, Pompei $P$, Ales KL, MacKenzie CR. A new method of classifying prognostic comorbidity in longitudinal studies: development and validation. J Chronic Dis 1987;40(5):373-83. 